\title{
Zfp-37 Is a Member of the KRAB Zinc Finger Gene Family and Is Expressed in Neurons of the Developing and Adult CNS
}

\author{
N. Mazarakis, ${ }^{*}$ D. Michalovich, A. Karis, F. Grosveld, ${ }^{1}$ and N. Galjart \\ M GC Department of Cell Biology and Genetics, Erasmus University, P.O. Box 1738, 3000 DR Rotterdam, The Netherlands; and \\ * Laboratory of Neurobiology, National Institute for Medical Research, The Ridgeway, Mill Hill, London NW7 1AA, United Kingdom
}

Received September 5, 1995; accepted January 18, 1996

The murine Zfp-37 gene encodes a protein with 12 zinc fingers at its C-terminus (Nelki et al ., 1990, Nucleic Acids Res. 18: 3655; Burke and Wolgemuth, 1992, Nucleic Acids Res. 20: 2827-2834). Contrary to the published data, our Northern blot analysis demonstrates not only that the Zfp-37 gene is expressed as 2.3, 2.6, and $4.2 \mathrm{~kb}$ mRNAs in testis, but also that there is a 3.7kb message in the adult mouse brain. Using a partial cDNA as a probe, we have isolated a brain-specific Zfp37 cDNA clone of $3.3 \mathbf{~ k b}$, whose sequence was extended to full length using $5^{\prime}$ end RACE. This revealed that the 3.7-kb mRNA is in fact a collection of transcripts with heterogeneous 5' ends. Comparison of CDNA and genomic sequences shows that the Zfp-37 gene is spread over a region of $\sim 20 \mathbf{~ k b}$ and consists of six exons, the large $3^{\prime}$ end exon containing the complete zinc finger domain, and 3' UTR. Our data show that the Zfp37 gene utilizes different promoters, alternative splicing, and differential polyadenylation to generate the distinct transcripts of brain and testis. Several protein isoforms are encoded by these mRNAs, some of which contain a truncated form of a conserved domain (Krüppel-associated box) found in other zinc finger genes. In situ hybridization analysis of postnatal brain sections indicates that the Zfp-37 gene is expressed in all neurons of the central nervous system. Together, these results suggest that ZFP-37 is a transcriptional regulator predominantly present in postmitotic cells from two different lineages. 1996 Academic Press, Inc.

\section{INTRODUCTION}

The zinc finger domain is one of a small number of nucleic acid binding motifs described to date (for a review see Harrison, 1991). It consists of 28-30 amino acids and contains four Cys and/or $\mathrm{H}$ is residues that

Sequence data from this article have been deposited with the EMBL/GenBank Data Libraries under Accession Nos. X89264X89266.

${ }^{1}$ To whom correspondence should be addressed. Telephone: $+31-$ 10-4087169. Fax: +31-10-4360225. coordinate the binding of a $\mathrm{Zn}^{2+}$ atom. In multi-zincfinger proteins of the $\mathrm{Cys}_{2} \mathrm{His}_{2}$ subclass, the individual fingers are often connected by a highly conserved linker of seven amino acids, which was first described in the Drosophila transcription factor and segmentation protein Krüppel (Schuh et al., 1986). A model was developed for these proteins in which each finger binds three nucleotides, with the C-terminus positioned on the $5^{\prime}$ side of the DNA recognition sequence and the $\mathrm{N}$-terminus facing the $3^{\prime}$ end (Nardelli et al., 1991; Pavletich and Pabo, 1991). Crystal structure determination of a number of zinc finger proteins bound to their cognate DNA sequences has shown this model to be incomplete, i.e., other modes of binding exist (Fairall et al., 1993; Pavletich and Pabo, 1993). Moreover, not every finger in a multi-finger protein will actually contribute to DNA binding (e.g., Keller and Maniatis, 1992), and the ZF motif can be used for RNA rather than DNA binding (Koster et al., 1991; Theunissen et al., 1992).

Mammalian genomes contain several hundred ZF genes (Bellefroid et al., 1989, 1993; Chowdhury et al., 1987) that can be further categorized on the basis of other protein motifs that they have in common. One of these is called the Krüppe-associated box (KRAB) (Bellefroid et al., 1991) or finger preceding box (FPB) (Rosati et al., 1991). This conserved domain is present in about a third of all $\mathrm{Cys}_{2} \mathrm{His}_{2}$-ZF proteins (Bellefroid et al., 1993) and may function as a transcriptional repression module (Margolin et al., 1994; Pengue et al., 1994; Witzgall et al., 1994).

Weare interested in identifying transcriptional regulators involved in neuronal differentiation pathways. We have focused our attention on the murine Zfp-37 gene, for which partial cDNA sequences derived from testis cDNA libraries have been published (Burke and Wolgemuth, 1992; Nelki et al., 1990). Both groups have shown that the Zfp-37 gene contains 12 zinc fingers, located at the $\mathrm{C}$-terminus of the protein and connected by the seven amino acid linker region, which is also found in Krüppel. Northern and Western blot analyses, as well as in situ hybridization and immunohistochemical data, suggested an exclusiverolefor the Zfp-37 gene 
in male germ cell devel opment (Burke and Wolgemuth, 1992; Hosseini et al., 1994). In contrast, we have found a brain-specific Zfp-37 transcript in adult mouse brain. We show here that this 3.7-kb mRNA is heterogeneous at its $5^{\prime}$ end and that it encodes various protein isoforms. Interestingly, some of these contain a truncated form of the KRAB domain. We also isolated a 35-kb genomic clone containing the complete Zfp-37 gene and determined its genomic organization. In situ hybridization on postnatal mouse brain sections confirms that Zfp-37 is expressed in the brain. More importantly, in the brain this putative transcriptional regulator is present in neuronal cells only, indicating that it might play a role in the development or maintenance of this cell type.

\section{MATERIALS AND METHODS}

Mice Mice used to prepare RNA for Northern blots, RACE, and $\mathrm{S} 1$ analyses were of C57/BL 6 or CBA origin. Mice used for making the brain CDNA library and in situ hybridizations were of the CBA/ CA or CBA/C57BL strains.

RNA isolation, Northern blot, and S1 analyses. RNA was prepared according to the method of Auffray and Rougeon (1980). Total RNA $(15 \mu \mathrm{g})$ was fractionated on $0.8 \%$ agarose gels containing $0.66 \mathrm{M}$ formal dehyde (F ourney et al., 1988). RNA was blotted and hybridized according to standard procedures (Sambrook et al., 1989). A 0.4-kb EcoRI fragment, labeled by random priming (Feinberg and Vogelstein, 1983), was used as a probe. RNA quality and loading were checked by staining the blot with methylene blue (Herrin and Schmidt, 1988). For S1 analysis, a genomic subclone, digested with BspEI, dephosphorylated, and subsequently digested with BamHI, was isolated from a low melting agarose gel and label ed at the BspE I site with T4 polynucleotide kinase (Sambrook et al., 1989). S1 experiments were performed with 10-20 ng of probe and $50 \mu \mathrm{g}$ of total RNA from brain, testis, kidney, or tRNA as published previously (Antoniou et al., 1988).

I solation of CDNA and genomic clones. The mouse brain CDNA library was constructed in Lambda ZAPII (Stratagene), as suggested by the supplier, using RNA from mice sacrificed at day of birth (P0). Approximately $1 \times 10^{6}$ unamplified phage were immobilized onto nitrocellulose filters, which were baked and hybridized as described above. A gel-purified EcoRI/Xbal fragment from the short testis cDNA (Nelki et al., 1990) was used as a probe. The filters were initially washed at low stringency, exposed wet to film, and then further washed and again exposed. Six strong and about 120 weaker duplicate double positives were detected with this procedure. Three strongly hybridizing clones (B2, B4, B6) were obtained after plaque purification rounds and were further characterized.

For the mouse genomic cosmid library, high-molecular-weight DNA ( $>200 \mathrm{~kb}$ ) was prepared from undifferentiated AB-1 embryonic stem cells (McMahon and Bradley, 1990). DNA was partially digested with $\mathrm{Mbol}$ and fractionated. Fragments larger than $30 \mathrm{~kb}$ were ligated into BamHI-digested pTCF cosmid arms (Grosveld et al., 1982). The ligation mix was packaged using Gigapack Gold, essentially according to the protocol of the supplier (Stratagene). A 0.4-kb E coRI and a 0.7-kb EcoRI/Xbal fragment, from the 5' and $3^{\prime}$ ends, respectively, of the B4 CDNA clone described above, were used to screen replica nitrocellulose filters (about $1 \times 10^{6}$ colonies). One clone (PKO 3.3.4) hybridized to both probes. It was colony purified and checked for rearrangements against mouse genomic DNA using Zfp-37-specific probes. Nucleotide sequences of CDNA and genomic clones were determined using the dideoxy chain termination method (Sanger et al., 1977) on double-stranded DNA (Murphy and Kavanagh, 1988). Conditions were modified for use with $\left[\alpha^{-35}\right.$ S dATP $\alpha S$ and Sequenase, as recommended by the supplier (USB). Sequence data were analyzed with the GCG (Devereux et al., 1984) and MacVector (Kodak IBI) software packages.

5' end RACE analysis. A modification of the original procedure for rapid amplification of CDNA ends (Frohman et al., 1988) was followed to obtain full-length Zfp-37 cDNAs. An oligonucleotide of 17 bases (complementary to nucleotides 347-364 in Fig. 2A) was hybridized to $100 \mu \mathrm{g}$ of total brain RNA from E14.5-day mouse embryos, and reverse transcription was carried out for $2 \mathrm{~h}$ at $41^{\circ} \mathrm{C}$ using super RT (HT Biotechnology Ltd.). Afterward, samples were passed through a centricon-100 filter (Amicon), washed, and dried on a speedvac. One-sixth of the material was used in a tailing reaction with dATP and calf thymus terminal transferase, according to the protocol of the supplier (Boehringer Mannheim). The reaction mix was then diluted $25 \times$ with water. Four percent of this sample was utilized in a first PCR (Saiki et al., 1988) with a Zfp-37-specific 3' end primer (18 bases, complementary to nucleotides 332- 349 in Fig. 2A) and two $5^{\prime}$ end primers, i.e., 5'-CTATCTAGAGAGCTCGCGGCCG-3' (a 22-mer adaptor) and the same sequence linked to $18 \mathrm{~T}$ 's (40-mer). After a first cycle at $95^{\circ} \mathrm{C}(5 \mathrm{~min}), 75^{\circ} \mathrm{C}$ (4 min, addition of Taq polymerase), $45^{\circ} \mathrm{C}$ ( $2 \mathrm{~min}$ ), and $72^{\circ} \mathrm{C}$ (15 min), 24 more cycles were performed at $94^{\circ} \mathrm{C}(45 \mathrm{~s}), 50^{\circ} \mathrm{C}(45 \mathrm{~s})$, and $72^{\circ} \mathrm{C}(2 \mathrm{~min})$ each. The PCR buffer composition was as suggested by the various suppliers. First round PCR material was diluted with water, and $0.1 \%$ was taken for a second round of PCR using a third Zfp-37-specific $3^{\prime}$ end primer (35-mer, complementary to nucleotides 156- 190 in Fig. 2A) and the $5^{\prime}$ end adaptor described above. Thirty cycles at $94^{\circ} \mathrm{C}(45 \mathrm{~s})$, $65^{\circ} \mathrm{C}(45 \mathrm{~s})$, and $72^{\circ} \mathrm{C}$ ( $1 \mathrm{~min}$ ) each were performed. After the PCR, samples were extracted with phenol:chloroform (1:1 v/v), passed over a centricon-100 filter, digested with proteinase K, extracted again, and ethanol precipitated. Samples were digested with Xbal and Pvull, gel purified, and subcloned. Nineteen subclones were se quenced and shown to be Zfp-37 derived.

In situ hybridizations. Mouse tissues were dissected and frozen immediately on dry ice. Sections (10 $\mu \mathrm{m})$ were cut, transferred onto slides, air-dried and fixed in 4\% paraformaldehyde. Slides were either used immediately or frozen at $-20^{\circ} \mathrm{C}$ for small periods of time prior to hybridization. In situ hybridizations were performed at $64^{\circ} \mathrm{C}$ as previously described (Nieto et al., 1992). An 800-bp E coRI subclone containing the $5^{\prime}$ end of the cDNA (Fig. 2A) and 300 bp of genomic upstream sequences were made into digoxigenin-labeled sense and antisense riboprobes, using T3 and T7 RNA polymerases and digoxigenin-11-UTP, as specified by the manufacturer (Boehringer Mannheim). Anti-digoxigenin antibodies, conjugated with alkaline phosphatase, were from the same supplier. Sections were mounted with Univert $(\mathrm{BDH})$.

\section{RESULTS}

\section{Brain-Specific Zfp-37 Expression and cDNA I solation}

Pilot Northern blot experiments indicated that Zfp37 is expressed in the adult mouse brain as well as in testis (not shown). We therefore screened a mouse brain CDNA library with a Zfp-37-specific probe and picked up three cl ones with different insert sizes. These were subcloned, and the longest (B4) was sequenced completely. A 0.4-kb EcoRI fragment from its $5^{\prime}$ end was next used to probe Northern blots with total RNA from various mouse embryonic and adult tissues. Only testis and brain express Zfp-37 at easily detectable levels (Fig. 1). Two highly abundant mRNAs of 2.6 and $2.3 \mathrm{~kb}$ and a minor transcript of $4.2 \mathrm{~kb}$ are present in testis, as reported previously (Burke and Wolgemuth, 1992; Hosseini et al., 1994). It is novel that a 3.7-kb message is easily detected in male and female mouse 


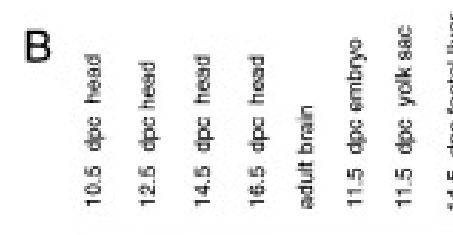

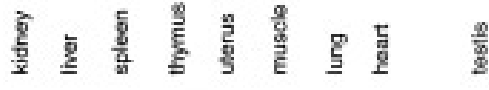
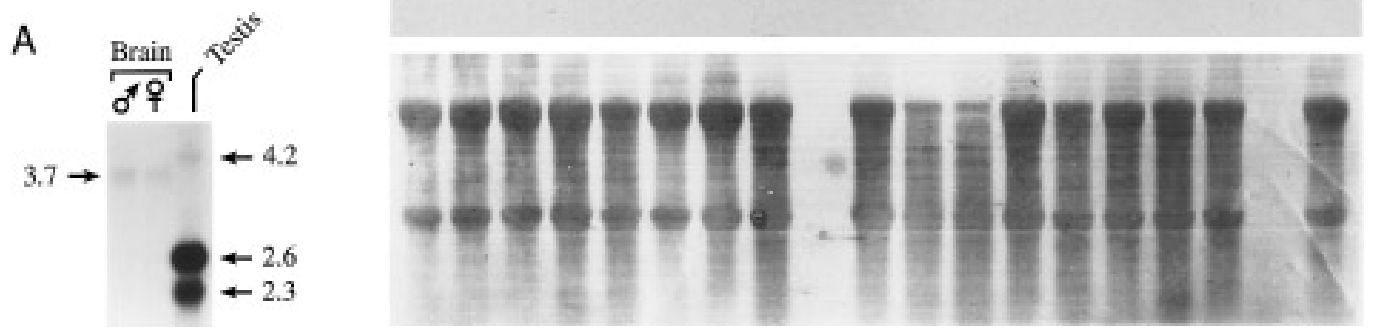

FIG. 1. Expression of Zfp-37 in embryonic and adult mouse tissues. (A) A Northern blot with $15 \mu \mathrm{g}$ per lane of total RNA from adult brain (male and female) and testis was probed with a 0.4-kb EcoRI probe, derived from the 5' end of Zfp-37 cDNA clone B4. After hybridization, the blot was washed and exposed to film for 1 week. Differences in length between the various Zfp-37 transcripts are well visualized on this blot. Sizes are indicated and were calculated using RNA markers (0.3- 7.4 kb). (B) (Top) A Northern blot with $15 \mu \mathrm{g}$ per lane of total RNA from various mouse tissues was hybridized as above. Exposure time was 3 days. The positions of the Zfp-37 transcripts are indicated. (Bottom) The same bl ot was stained with methylene blue to measure quantity and quality of RNA. N ote the slight degradation in liver and spleen samples.

adult brains (Fig. 1) and that low levels of this transcript are visible in heart, lung, uterus, thymus, liver, and kidney on long exposures of the autoradiograph (not shown). The 3.7-kb message is readily detectable in the developing head region from Day 10.5 of gestation onward (Fig. 1B). We have not tested whether the Zfp-37 gene is expressed earlier in devel opment. Probes derived from the zinc finger region gave similar results, as presented in Fig. 1, although additional weak signals around 1.4 and/or $2.4 \mathrm{~kb}$ were occasionally observed (not shown). Thus, our data show more than the previously published results (Burke and Wolgemuth, 1992; Hosseini et al., 1994), probably because of the use of more sensitive probes in our case. Therefore, the most abundant expression of Zfp-37 in the adult mouse is in testis, with a more moderate expression in the brain. Very low levels of transcript are detected in a number of other tissues.

The size of the longest cDNA insert (B4) was $3.3 \mathrm{~kb}$, which appeared too short to account for a 3.7-kb mRNA. Therefore, a 5' end RACE analysis was performed to obtain a full-length CDNA. This yielded three different $5^{\prime}$ ends. Most of the RACE clones (13/19) are of the socalled type Ia (Fig. 2A). Type I a clones differ internally in that regions of nine and/or three nucleotides are present in some clones, whereas they are absent in others. As shown below, this is due to alternative splicing. Note that these insertions do not cause a frameshift in the putative open reading frame (ORF). The sequences of the other two groups of $5^{\prime}$ end clones, called I b and II, are shown separately in Figs. 2B, and $2 \mathrm{C}$, respectively. Thus, the 3.7-kb Zfp-37 mRNA is heterogeneous at its $5^{\prime}$ end. RT-PCR with novel sets of primers specific for exon Ia or Ib verified that both exons are present in Zfp-37 mRNAs from adult brain and testis (not shown).

Comparison of the published Zfp-37 sequences with the brain-specific cDNAs reveals several interesting facts. First, the $5^{\prime}$ ends of brain- and testis-derived cDNAs differ completely in sequence from nucleotide position 131 in Fig. 2A (asterisk) going upstream. When a type la sequence is linked to the B4 CDNA, the ORF proposed by others (Burke and Wolgemuth, 1992) is extended toward the $5^{\prime}$ end. Instead of ATG $_{209-211}$, it is now ATG $_{86-88}$ that would be first encountered by the scanning ribosome. Assuming translation starts at this first ATG, protein isoforms of approximately 67 $\mathrm{kDa}$ are produced. These differ at their $\mathrm{N}$-termini by one, three, or four amino acid residues, due to the variability in type la sequences that we have described 
(AT) A TCG GCC TCT GAC TCC ATC GAG GAC CTG ACA AAG CCA GAG GCG CTG GAT GGG AGG AGT GCG GAC AAA GCC AGG CGT CCG GAG GAG ATG

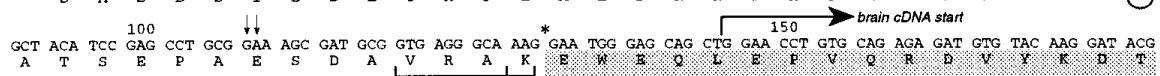

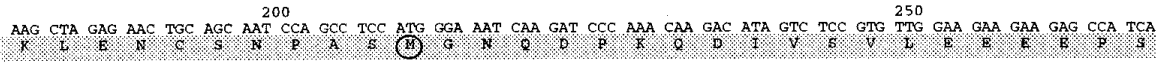

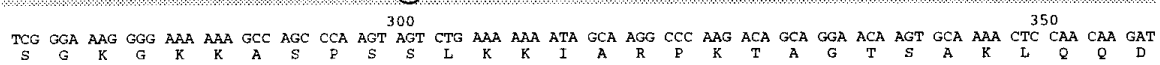
GAT GAG CAT AGG GAG GAA AAG CAG AAG TCC CAA AGC AAA 400 CTT ACT AAG GAA GTG ACA CTC AGG AAG AAA AGT TCC AAC AGC AAG AAA AGC

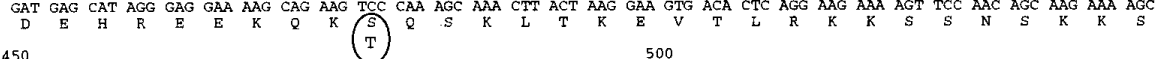

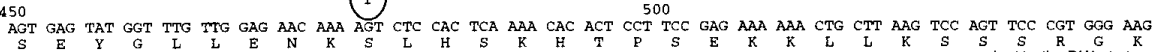

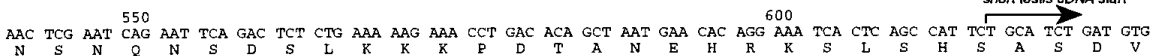

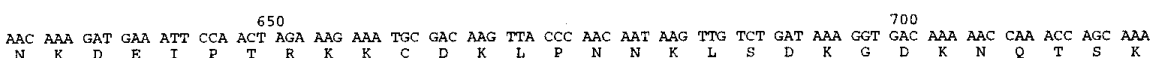
N 1500000

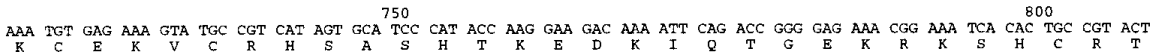

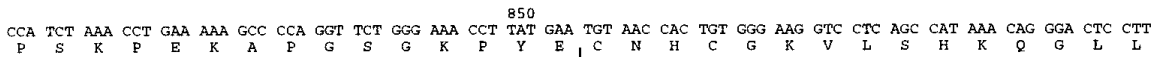
9 GAC CAT CAA AGA ACT CAC ACT GGG GAG AAA CCA TAT GAA TGT AAT GAA TGT GGG ATA GCT TTC AGC CAG AAG TCC CAC CTT GTT CTA CAT

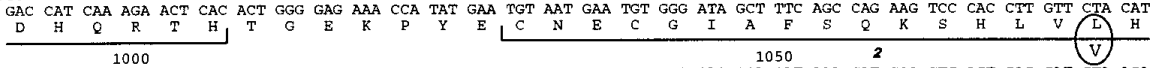

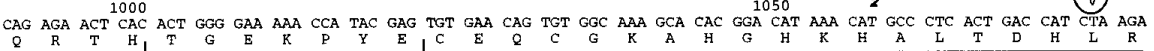
\begin{tabular}{lllllllllllllllllllllllll}
$Q$ & $R$ & $T$ & $H$ & $T$ & $G$ & $E$ & $K$ & $P$ & $Y$ & $E$ & $C$ & $E$ & $Q$ & $C$ & $G$ & $K$ & $A$ & $H$ & $G$ & $H$ & $K$ & $H$ & $A$ & $L$ \\
\hline
\end{tabular} ATC CAT ACT GGA GAA AAG CCCC TAC AAA TGT AAT GAA TGT GGC AAA ACG TTTT AGA CAC AGC TCA AAC CTTT ATG CAA CAC CTA AGA TCT CAC ACG GGT GAG AAG CCG TAT GAA TGT AAG GAA TGT GGC AAA TCC TTT AGA TAT AAT TCA TCT CTT ACT GAA CAT GTG AGA ACA CAC ACA GGT

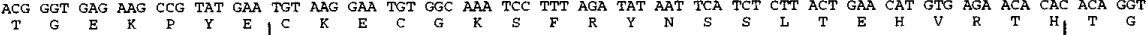

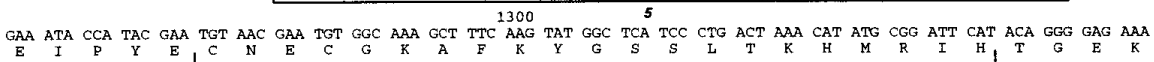

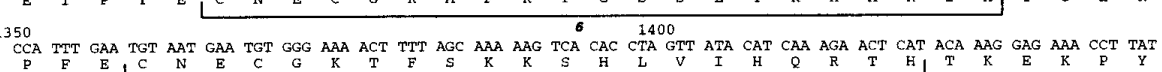

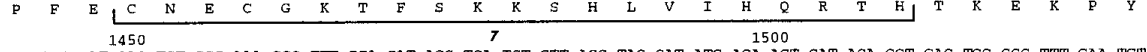

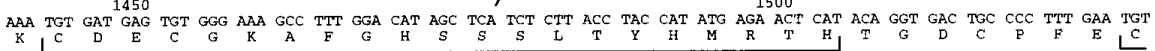
155001600 CCC TAT GAG TGT GTT GAA

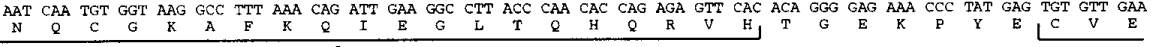
TGT GGG AAA GCC TTT AGT CAG AAG TCA CAC CTC ATC GTA CAC CAG AGA ACT CAT ACA GGG GAG AAA CCC TTT GAA TGT TAT GAG TGT GGA

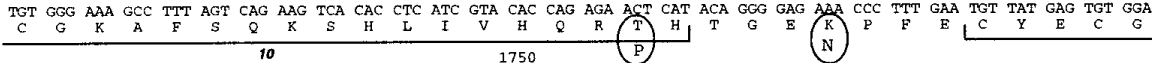
AAA GCC TTC AAT GCA AAA TCA CAA CTT GTT ATT CAT CAG AGA TCC CAC ACT GGA GAG AAA CCC TAT GAA TGT ATT GAA TGT GGT AAA GCC

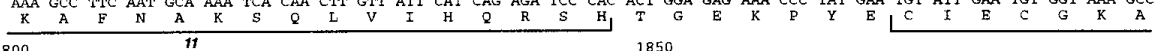

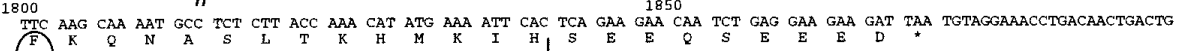
$\begin{array}{lll}V & 1900 & 12 \\ \text { GTTGGTATTATPTAACCTTAAAGATGTTCTCAATTTGATGATGTTAGAATATCTTTTTTTAGGAAATCATTCCTGGTGATACATGAGAGAATTTGAATATGGATCTTTACATAAGATGG }\end{array}$

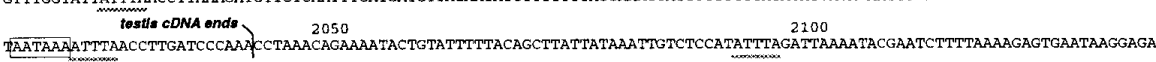
2150
TATCATAATCAGTAAAGTGCTTGTTAAGCAAGTACAGAGATCTGAATTCCATCCCTAAGGATTGATAAAAAGCTAGATGTGGGGGCATGCTCTTGCAATCTCACAATTGGGAAAGTTGA 2300
ACAGGAGAGTCCTGAGAACTCCTGACCAACCAGACTCATTPAATPTGCAAGCCATAGGTCACAAGAAAAAAGAACTGTTTTAAAAATAGGGTAGAGGGTTCCTGAGAAACAGTACTCAAG 2400
GTTGACACATGTACACACACAGACACACACAGACACACAGACACACACACAGACACACACAGATACACACACACACACAGACACACACACACAGACACACACACAGTTGTGAaAaTGTTA

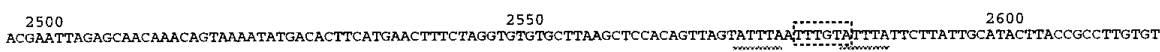
2650
2700
AAAATACAAGTGAGGGTGAGGGTATCCTCTGGTATTGTCTAGCTTTCTTTGTTAATGTCTCTGTTAGCTTCTGAATTAGTAGAAACTTTCGGAGTTGTTTTGTTTTGTITIGAGACAAT 2750
ATCTCACCATATAGTCCTGGTAGCCTGGAACTTGTTACGTGTACCAGGCTGGCCTTGAACTTGTTATGTAGACCAGGCTGACCTTGAACTCACAGAGATATGTCTGTCTCTECCTCCCA

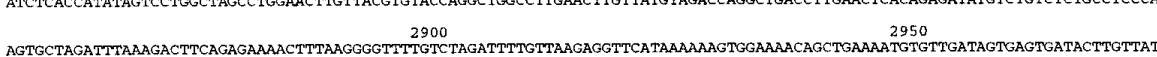
AGTGCTAGATTTAAAGACTTCAGAGAAAACTPTAAGGGGTTTTGTCTAGATTTTGTTAAGAGGTTCATAAAAAAGTGGAAAACAGCTGAAAATGTGTTGATAGTGAGTGATACTTGTTA ATTGTTAAGTATAGAAAATGGATTGTTAAACCATCTATATAGTATGTTCTCATTGTGGACAGAGTGTGTATGTGTGTCCCTGTACTCTGTGTACATATATATGTGTTTATCCCCATATA: 3150
...3190
. TTGTATATGAATAGACTTTTTTPAACCTATAAGAAAATTAATGGTGGCTTCCACTGAACACAAGAGTGCAGCATTGAGGTCTGACATGTTTATCATGTACATGTGTCTAATTCAAATATG 3300
TATTATATAAAAATACATTCATATGTGCAAATTACACAATTTAGAACAATAGCTGAGTCTATTTCACAGTGTTATTTGAAAAAGTGACATAAGATTTCTATGCTCCTTTATGAACAATG

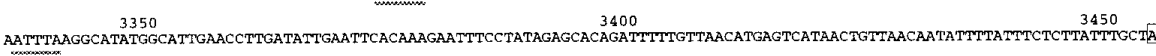
TTAAAAACTAAAGATGAATGAAAAAAAAAAAAAAAAAA

C TITGAGAATCTGTGGCGTTGTTGTPGAATAGTCTTTTGGGAAGGCCGGTGTTCTAAGGGA CCAAGGTATTGGGGTATTATGGGCATATGGAGTATTAAGTGCCCGTGATATMGGGGGCAT
ATGGAGTTGGGGATTGCTGTAACTTTCGGAAATCAGTATTTTGACGGATCTGTCTAGGAA
TGGGAGCAG

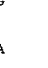

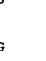


above. Since we cannot formally exclude the possibility that translation initiation takes place upstream of the first ATG, for example at a non-AUG codon, we have indicated the putative translated residues upstream of $A T G_{86-88}$ in italics in Fig. 2A. If the Zfp-37 mRNAs start with type Ib or type II sequences, in-frame stop codons upstream of $\mathrm{ATG}_{209-211}$ are present. As described by Burke and Wolgemuth (1992), only a single protein of 553 amino acids $\left(M_{r} 62.6 \mathrm{kDa}\right)$ would then be produced.

The first published Zfp-37 testis cDNA (Nelki et al., 1990) contains an insertion in the sequence that leads to a frameshift and an incorrect $\mathrm{N}$-terminal domain. This mistake was discussed previously (Burke and Wolgemuth, 1992). Several other base differences are found when the various Zfp-37 coding regions are compared, some of which cause amino acid alterations (circled residues in Fig. 2A). The mutations at nt 386 and 983 could represent polymorphisms; the others (nt 1667, 1684, and 1799) are probably mistakes in the published testis cDNAs.

Thelong 3' UTR of the B4 CDNA has several noteworthy features. It contains seven AUUUA elements in addition to the two present in the testis CDNA $3^{\prime}$ UTRs (underlined in Fig. 2A). These sequences could shorten the half-life of Zfp-37 mRNAs, as seen in other transcripts (for review see Decker and Parker, 1994). The 3' UTR also contains two putative immediate response boxes at positions 2577- 2582 and 3094- 3099 (IRBs; dotted boxes in Fig. 2A). These are DNA regulatory elements, which mediate the response to PDGF in the immediate-early J E gene (Freter et al., 1992). IRBs are found in the $3^{\prime}$ ends of many immediate-early genes. Like Zfp-37, some of these genes contain an incomplete IRB (6/7 bases correct). Finally, the B4 cDNA 3' UTR contains a CA-dinucleotide repeat of about 100 bases.

All ZFP-37 isoforms have 12 zinc fingers of the $\mathrm{Cys}_{2} \mathrm{His}_{2}$ type located at their C-termini. The fingers are connected by the Krüppel-like amino acid linker. A short, acidic tail of nine amino acids follows the last finger. The N-terminal portion of ZFP-37 is rich in charged/polar amino acids and contains a degenerate zinc finger motif near the first real finger. Such "rudi- ments" are also found in other members of this class of proteins (Attar and Gilman, 1992; Bellefroid et al., 1993). Further inspection of the sequence reveals that ZFP-37 is actually a member of the KRAB/FPB family of zinc finger proteins (Bellefroid et al., 1991; Rosati et al., 1991); i.e., the type Ia cDNAs encode an almost complete KRAB domain, whereas the other CDNAs code for isoforms with only the degenerated " $B$ " region. Together, our data suggest that the Zfp-37 gene encodes various protein isoforms of the KRAB multi-zincfinger family.

\section{Organization of the Zfp-37 Gene}

The complicated structure of the Zfp-37 cDNAs prompted us to determine the genomic organization of this gene. The Zfp-37 gene could be isolated on a single cosmid clone. It covers roughly $20 \mathrm{~kb}$ and is located toward the $3^{\prime}$ end of the $35-\mathrm{kb}$ cosmid insert. Sequence analysis of genomic subclones and comparison to Zfp37 cDNAs suggests that the gene contains six exons, called Ia, I b, It, II , III, and IV (Fig. 3A). Exons Ia and Ib are colinear with the 5' RACE clone types Ia and Ib, respectively. Exon It (t stands for testis) was found downstream of exon I $\mathrm{b}$ and comprises 17 bases. These nucleotides align perfectly to the extreme $5^{\prime}$ end (without the first 7 bases) of one of the published Zfp-37 testis cDNAs (Burke and Wolgemuth, 1992) and are followed by a putative splice donor in the genomic sequence (Fig. 3B). The first seven nucleotides of the testis CDNA (Burke and Wolgemuth, 1992) are LambdaZAP-derived.

The 5' end RACE analysis suggests that transcription of the Zfp-37 gene starts from three different promoters and that some of the primary transcripts are alternatively spliced. We have summarized these putative events in Fig. 3C. The major transcription initiation site(s) (11/19 RACE clones start here) is 5' to exon Ia. The occurrence of type Ib (2/19) and type II (4/19) RACE clones suggests that transcription can initiate at two other promoters, one just downstream of exon Ia and the other $5^{\prime}$ to exon II. Alternative splicing of exon Ia to exon II occurs at two donor sites, which are

FIG. 2. Brain-specific Zfp-37 cDNA sequences. (A) Composite sequence of $5^{\prime}$ end RACE-derived sequences linked to the 3.3-kb mouse brain CDNA clone (B4), isolated from a PO CDNA library. The RACE analysis yielded three types of clones; the most common one (type Ia; $13 / 19$ clones) is linked to B4. Most type la clones start 2 nucleotides apart (brackets at $5^{\prime}$ end). Two shorter type Ia clones start at nt 107 and 108 (vertical arrows). The $5^{\prime}$ ends of the B4 CDNA and the short testis clone are marked, as is the $3^{\prime}$ end of the testis CDNAs (Burke and Wolgemuth, 1992; Nelki et al., 1990). The point of 5' end divergence between long testis cDNA (Burke and Wolgemuth, 1992) and brain cDNAs is marked by an asterisk. The two potential polyadenylation sites (solid lines) and IRBs (dotted lines) are boxed. The nine AUUUA elements are underlined (wavy line). Amino acid numbering is indicated to the right. The first two methionine residues are circled. The amino acid sequence upstream of the first methionine is italic to indicate that it may be translated. Alternatively spliced amino acid residues (VRA/K) are underlined, as are the 12 zinc fingers at the C-terminus of ZFP-37. Amino acid substitutions due to differences in the various sequences are circled. The KRAB domain is shaded. (B) Two type Ib cDNA clones derived from the 5' end RACE analysis. The sequence of the longer clone is given; the sequence of the second clone is colinear with the first (dotted underlining) up to the vertical arrow (splice site). The shorter clone has an insertion of 3 nucleotides at this position. Downstream of the arrow, cDNA clones become colinear. In the long clone, the presence of three short ORFs is indicated. (C) Four type II clones derived from the 5' end RACE analysis. The sequence of the longest clone is given; the others are indicated with dotted lines. Vertical arrows mark splice acceptor sites of exon II (see below). 
A

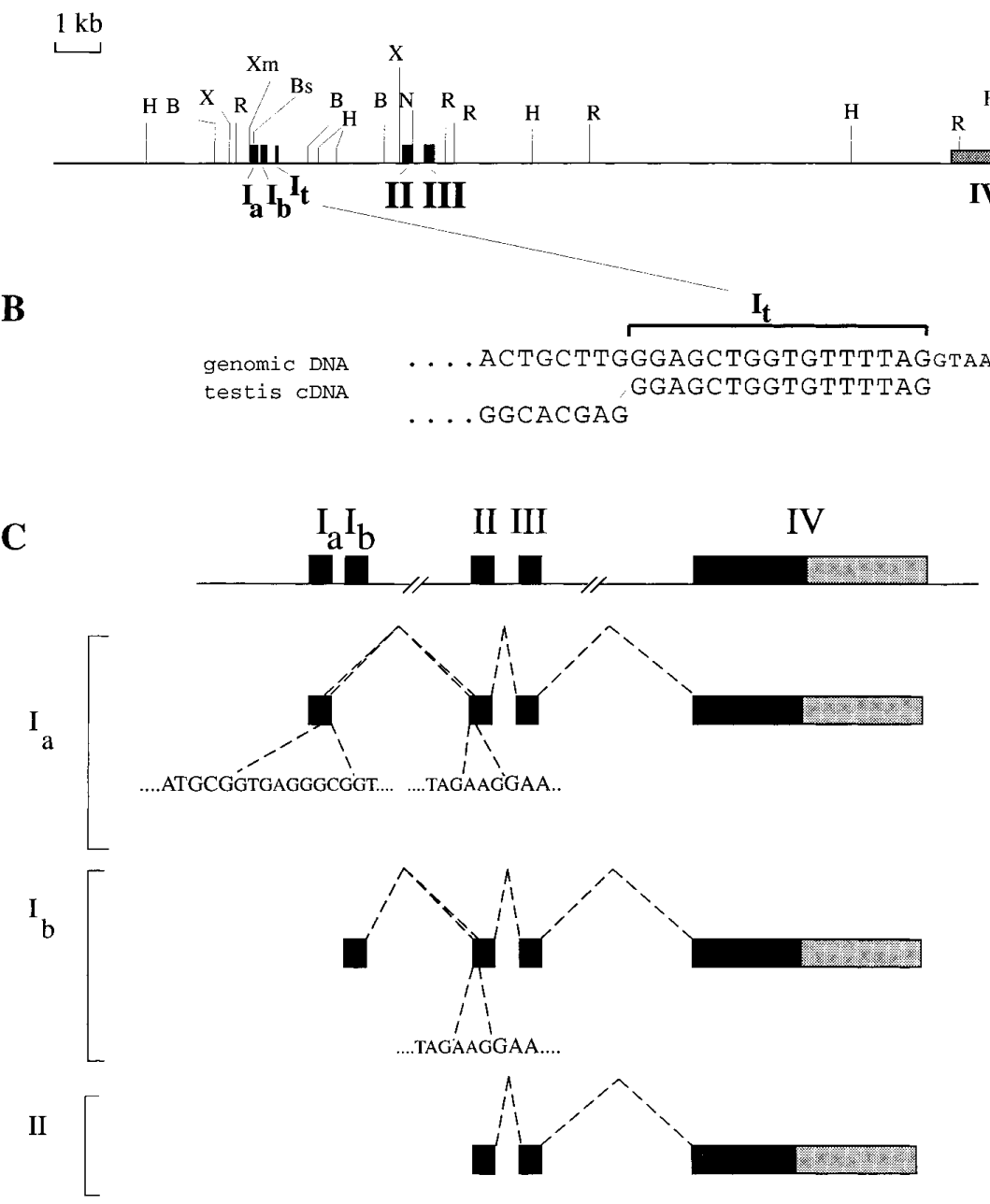

FIG. 3. Genomic organization and transcription pathways of the Zfp- 37 gene. (A) A cosmid with an insert of $35 \mathrm{~kb}$ was isolated from a mouse genomic library. The Zfp-37 gene is located toward the 3' end of this insert, on an area of about $20 \mathrm{~kb}$. A partial restriction enzyme map of this region is shown. The six exons are Ia, Ib, It, II, III, and IV; their positions are indicated by boxes (nucleotide positions of exonintron boundaries within the Zfp-37 CDNA can be found in the EMBL database). Exon IV contains the 3' UTR (shaded box). H, HindlII; B, BamHI; X, Xbal; R, EcoRI; Xm, Xmal; Bs, BspEI; N, Ncol. (B) The 5' end of one of the two testis cDNAs (Burke and Wolgemuth, 1992) was found to align perfectly to a genomic sequence of 17 bases, which was therefore termed exon It (t stands for testis). The first seven nucleotides of the CDNA were not homologous to the genomic sequence and were later found to be lambda-ZAP-derived. At the $3^{\prime}$ end of exon It there is a splice donor site, and the remainder of the testis CDNA aligns to exons II, III, and IV. This makes it likely that exon It is genuine, but its $5^{\prime}$ border has yet to be defined. (C) Putative Zfp-37 transcription initiation sites and modes of alternative splicing of Zfp-37 transcripts in the brain. The 5' end RACE clones map to three different positions within the gene, suggesting that transcription initiation takes place in front of exons Ia, Ib, and II. Splicing of exon Ia to exon II occurs via two splice donor and two splice acceptor sites located 9 and 3 nucleotides apart, respectively (see sequences below the Ia-II splicing pathway). Splicing of Ib to II makes use of the same two acceptor sites.

nine nt apart, and at two splice acceptor sites, which are three nt apart. Splicing of exon Ib to exon II again makes use of the two acceptor sites at the $5^{\prime}$ end of exon II (Fig. 3C).

Preliminary sequence analysis of a genomic 450-bp region $5^{\prime}$ to and covering exon Ia shows it to be GCrich (66\%) and to contain 40 CpG dinucleotides (not shown), indicative of a CpG island (Bird, 1986). These usually colocalize with the $5^{\prime}$ end of genes, suggesting that the composite CDNAs depicted in Fig. 2A are full length. This was confirmed by S1 nuclease protection with a genomic fragment of $870 \mathrm{bp}$, labeled at a BspE I site (position 79, Fig. 2A) and covering the complete CpG island and upstream sequences. Theresults of this experiment show two sites of transcription initiation in the brain (arrows in Fig. 4), one of which colocalizes with the $5^{\prime}$ end of thetype I a cDNA clones. In the testis, transcription initiation is spread over an area of more than 200 bases, with the bulk taking place some 250 bases upstream of the brain sites (bracketed in Fig. 4). S1 analysis with a probe closer to the testis transcription initiation sites gives similar results (not shown). 


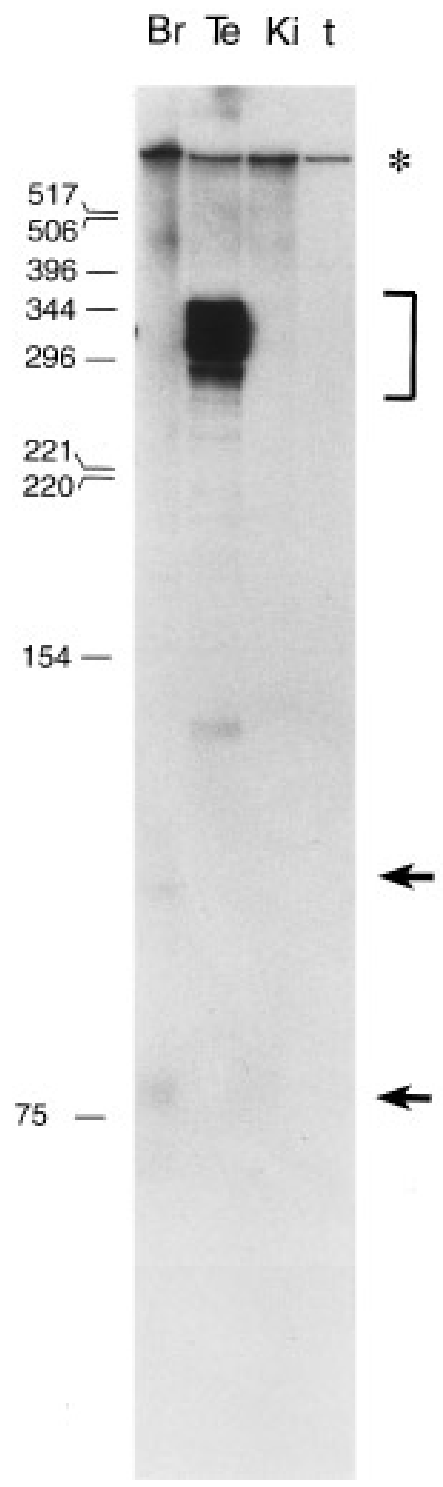

FIG. 4. Zfp-37 transcription initiation at exon la as measured by $\mathrm{S} 1$ analysis. A genomic probe spanning the region from the BspE I site in exon la toward a more 5' BamHI site (Fig. 3A) was used in a S1 nuclease protection experiment with $50 \mu \mathrm{g}$ of total RNA from brain (Br), testis (Te), kidney (Ki), or tRNA (t). S1-protected fragments were separated on a sequencing gel (marker DNA sizes are indicated to the left). The probe is visible in all lanes (asterisk). Multiple transcription initiation sites are present in testis (bracket), whereas there are only two weakly visible sites in brain (arrows).

Thus, transcription initiation from within the presumptive CpG island gives riseto ZF P-37 isoforms with the KRAB-A domain. Initiation outside the CpG island yields isoforms without this region.

At the $3^{\prime}$ end of exon IV, the genomic sequence is colinear with the different cDNAs until the start of the respective $\operatorname{poly}(A)$ stretches, demonstrating the presence of two polyadenylation sites in the Zfp-37 gene. Preliminary experiments with a probe between these two sites fail to show the 2.6- and 2.3-kb messages, whereas the 3.7-kb mRNA is detected (not shown).
Therefore, the first of the two polyadenylation sites is predominantly used in testis, whereas the second is predominantly used in brain.

\section{Expression of Zfp-37 in Mouse CNS}

In situ hybridization was used to examine the spatial distribution of Zfp-37 in the developing embryo at 12.5 d.p.c. and in postnatal mouse brains. In the E $12.5 \mathrm{em}-$ bryo, the strongest hybridization is observed in the germinal neuroepithelium, predominantly in the diencephalon, mesencephalon, and myelencephalon, although structures outside the CNS are also positive (Fig. 5A). The signal is present in the ventricular zone, indicating that the gene is expressed in multipotent precursor cell populations (Figs. 5C and 5D). In the neonatal brain (P3, P6, and P15), Zfp-37 is expressed by a subset of neurons in the developing cerebellum (Fig. 5J ), hippocampus, and neocortex (not shown). In the cerebellum, stronger hybridization is observed in Purkinje cells than in the granular layer at these early stages of development.

In adult brain, Zfp-37 expression appears to be panneuronal (Figs. $5 \mathrm{E}-5 \mathrm{H}$ and $5 \mathrm{~L}-5 \mathrm{~N}$ ), although the differential hybridization within the various neuronal populations is still observed. In the hippocampus, there is intense hybridization with pyramidal neurons and dentate granules (Figs. 5E - 5G), but little or no hybridization in regions where glia are present, such as in the fimbria in the hippocampus, suggesting either that this gene is exclusively expressed in neurons or that it is also expressed in glia but at very low levels. In the olfactory bulb, Zfp-37 expression is observed in the mitral and granule cell layers (Fig. 5N). Strong hybridization is also observed throughout the cortex (Fig. 5I). The hybridization signal is mainly cytoplasmic, but nucleoli are also stained (Fig. 5G). Parallel control experiments with the sense probe were negative (Figs. $5 \mathrm{~B}$ and $5 \mathrm{~K}$ ), except for a weak nucleolar staining in every cell. These data suggest that Zfp-37 is on early in precursors that give rise to the nervous system and that as the brain develops, Zfp-37 expression becomes restricted to the neuronal but not the glial cell population. In neurons, Zfp-37 expression is differentially regulated within distinct populations. Zfp-37 expression is not exclusive to neurons, since the Northern blot data show that Zfp-37 is also present outside the nervous system in both embryonic and adult organisms.

\section{DISCUSSION}

The Zfp-37 gene was previously shown to encode a protein with 12 zinc fingers at its C-terminus. Two testis Zfp-37 cDN As have thus far been published, a short truncated one of $1.4 \mathrm{~kb}$ (Nelki et al., 1990), which contains a sequencing mistake leading to an ORF with an incorrect N-terminal domain, and a longer clone of 1.9 kb (Burke and Wolgemuth, 1992), which was postu- 

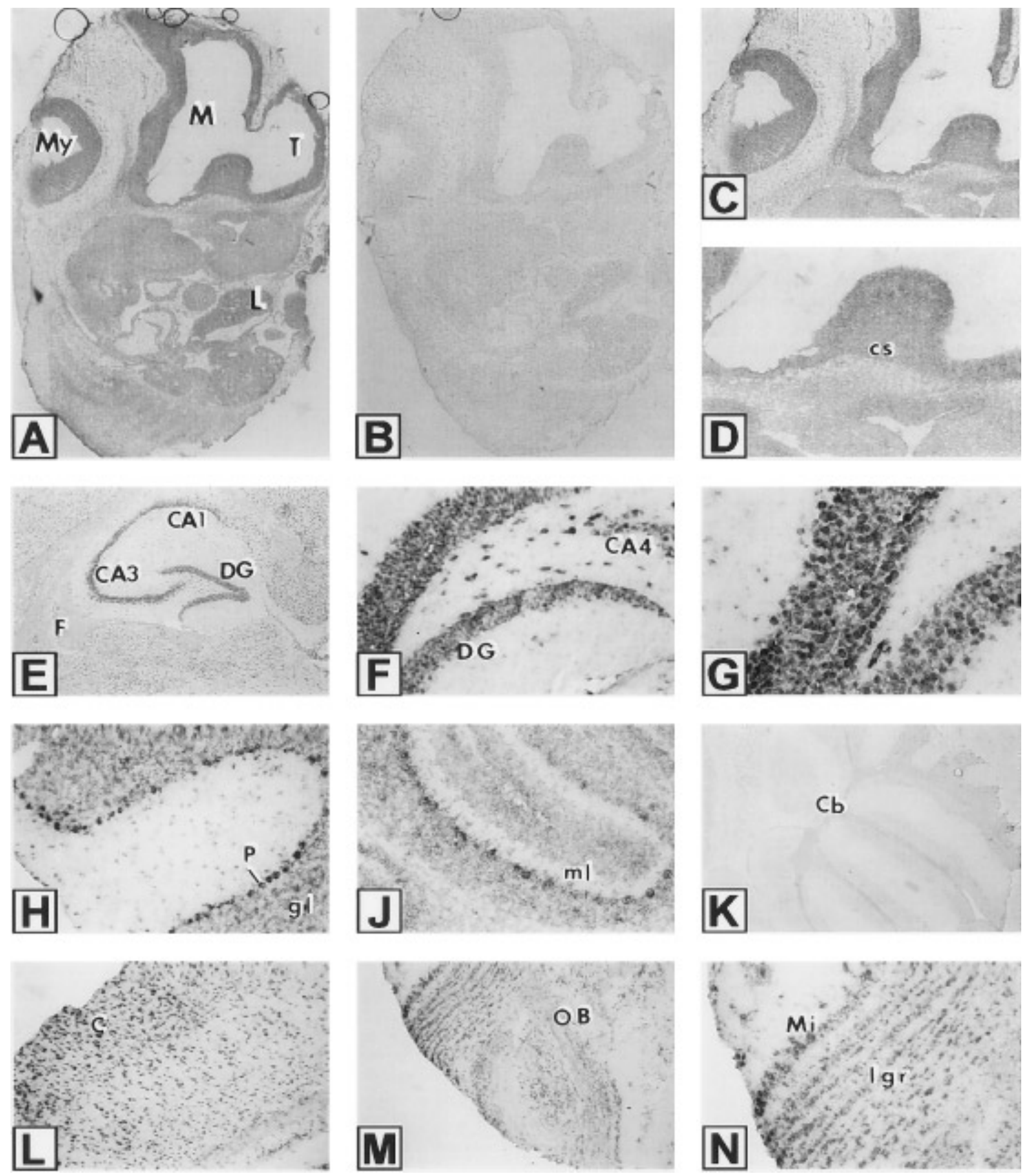

FIG. 5. In situ hybridization analysis of Zfp- 37 in mouse embryo and adult mouse brain. Frozen tissue sections of $10 \mu \mathrm{m}$ were fixed on slides and hybridized with digoxigenin-labeled riboprobes. Label was visualized using anti-digoxigenin antibodies conjugated to alkaline phosphatase. (A) E12.5-day embryo, antisense probe. The most intense signal is observed in ventricular layers of the head (germinal neuroepithelium), while there is also some hybridization above background in the rest of the trunk. (B) Same stage embryo, sense probe. Only a general background labeling is observed. (C) Magnified picture of E12.5-day embryo showing the hybridization of the antisense probe around the third and fourth ventricles. (D) Magnification of the presumptive striatum in the same embryo. (E) Adult hippocampus, antisense probe. Strong hybridization in the pyramidal and dentate granule neuronal Iayers. Little hybridization is present in the fimbria, suggesting neuronal specificity for the signal. (F) Labeled dentate granules (10X). (G) Labeled dentate granules (20X). (H and J) Adult and P6 cerebellum, antisense probe, showing strong hybridization of Purkinjeneurons and weaker staining of granules. (K) Adult cerebellum, sense probe. (L) Cerebral cortex, antisense probe. (M) Olfactory bulb, antisense probe. (N) Magnification of olfactory bulb, showing hybridization in mitral cells and internal granules. T, telencephalic vesicle; $M$, mesencephalic vesicle; My, myencephalic vesicle; $L$, liver; cs, corpus striatum; F, fimbria; CA1, CA3, CA4, pyramidal fields of hippocampus; DG, dentate granule layer of hippocampus; P, Purkinje cell layer; gl, granular layer of cerebellum; ml, molecular layer of cerebellum; Cb, cerebellum; C, cortex; OB, olfactory bulb; Mi, mitral cells; Igr, internal granular layer. 
lated to be full length and to give rise to a protein with a $\mathrm{M}_{\mathrm{r}}$ of $62 \mathrm{kDa}$. In the adult mouse, Zfp-37 expression was detected only in testis, with the mRNA being localized to round and elongating spermatids. ZFP37 was therefore speculated to be a transcriptional regulator of spermiogenesis (Burke and Wolgemuth, 1992). We present here the cloning and characterization of brainspecific Zfp-37 cDNAs as well as the isolation of a cosmid of $35 \mathrm{~kb}$ containing the complete Zfp-37 gene. Three experimental approaches, i.e., Northern blot analysis, RT-PCR, and in situ hybridization, all confirm that the Zfp-37 gene is expressed not only in testis but also in brain. We suggest that the composite cDNAs described in this paper are full length and represent the 3.7-kb Zfp-37 transcript found on Northern blots. As far as the other transcripts that appear to be testisspecific are concerned, S1 analysis demonstrated that in the CpG island, testis transcription initiates roughly 250 bases upstream of brain transcription. mRNAs generated at one of these upstream sites and identical to the $3.7-\mathrm{kb}$ message until the first polyadenylation site would have predicted lengths of about $2.6 \mathrm{~kb}$, if a poly(A) tail of 300 bases is included in the calculation. This size correlates well with the $2.6-\mathrm{kb}$ message found in testis. The 1.9-kb testis cDNA published previously (Burke and Wolgemuth, 1992) could represent the 2.3$\mathrm{kb}$ mRNA found on Northern blots, but its transcription start needs to be mapped. Finally, the $4.2-\mathrm{kb}$ mRNA probably represents another zinc finger gene (data not shown).

Those Zfp-37 mRNAs containing exon I a code for protein isoforms of about $67 \mathrm{kDa}$, which is $5 \mathrm{kDa}$ more than was predicted previously (Burke and Wolgemuth, 1992). A KRAB domain is present in these ZFP-37 isoforms (Bellefroid et al., 1991; Rosati et al., 1991). The KRAB domain has been divided into a highly conserved $A$ region and a degenerate $B$ region. In vitro studies suggest that the KRAB domain acts as a DNA binding dependent repressor of transcription and that the $A$ region is necessary and sufficient to obtain this effect (Margolin et al., 1994; Pengue et al., 1994; Witzgall et al., 1994). Obviously, the functional significance of the ZFP-37 KRAB region needs to be established, but its presence suggests that ZFP-37 is a DNA rather than a RNA binding protein. In this context, it is noteworthy that fingers 2 and 10 of ZFP-37 are almost identical and could bind the same sequence. In contrast, fingers 1 and 3 have amino acid substitutions in a conserved phenylalanine ( $F$ to $L$ in finger $1 ; F$ to $H$ in finger 3 ). This could compromise their capacity to bind nucleic acid.

Expression of ZFP-37 isoforms is controlled at various levels. First, transcription of the gene appears to start in three regions, with the bulk of transcripts originating in the presumptive CpG island in which exon Ia is located. The neuronal nitric oxide synthase (nNOS) genealso has two closely linked promoters, like the Ia and Ib presumptive promoters of Zfp-37, and one nNOS promoter is embedded in a CpG island (Xie et al., 1995). The functional significance for Zfp-37 is that mRNAs initiated at the CpG island encode ZFP. 37 isoforms with a KRAB domain, whereas the other transcripts do not. Thus, a switch in promoter usage leads to the production of different proteins. This is not the case for nNOS.

A second level of control of expression could be mRNA stability, as suggested by the presence of nine AUUUA elements in the 3' UTR of the Zfp-37 gene. Turnover of steady-state levels of 3.7-kb Zfp-37 mRNAs may be much higher than that of 2.6- and 2.3-kb transcripts, which have only two AUUUA elements in their $3^{\prime}$ UTRs. A very similar situation exists in the CREM gene, which was shown to be highly expressed in testis compared to brain due to nine AUUUA elements in the brain 3' UTR (Foulkes et al., 1993). The presence of these elements and of two incomplete IRBs (Freter et al., 1992), indicates that Zfp-37 could be a member of the immediate-early gene family.

In addition to control exerted at thelevel of transcription and mRNA stability, regulation of mRNA translation is yet another means by which ZFP-37 protein levels may be kept in check within a cell. In the case of the type Ib Zfp-37 mRNAs, ribosome translation at the correct ATG (209-211) can only take place if three short upstream ORFs are first bypassed. It has been shown for the yeast transcription factor GCN4 that such downstream initiation only occurs when cells are starved (Hinnebusch, 1994). Therefore, it will be particularly interesting to follow the expression of type $\mathrm{Ib}$ Zfp-37 mRNA; perhaps it can be linked to a specific cellular event or to a subtype of cells.

Together, the data suggest a model of ZFP-37 function in which the expression of the gene is carefully regulated at the level of transcription as well as translation. It is tempting to speculate that the different protein isoforms fulfill functions that have to do with the regulation of transcription of genes in postmitotic cells, since Zfp-37 expression in the adult mouse is most abundant in round and elongating spermatids and in neurons. It remains to be determined whether ZFP-37 function is the same in both tissues and throughout development and whether the protein is a transcriptional activator and/or repressor. It could also be that Zfp-37 gene products are involved in maintaining higher order chromatin structure, for example the opening or closing of gene loci. This would resemble the insulator function of suppressor of hairy wing, $\mathrm{Su}(\mathrm{Hw}$ ) (Roseman et al., 1993), a protein with 12 zinc fingers found in Drosophila melanogaster (Parkhurst et al., 1988).

\section{ACKNO WLEDGMENTS}

We thank Dr. Daniel Nelki for providing the 1.4-kb Zfp-37 testis cDNA, Vangelis Stavropoulos for assisting with the subcloning and sequencing of the brain Zfp-37 cDNA clones, Ton Verkerk for per- 
forming the RT-PCR, Mirko Kuit for photographic work, and Drs. Angela Nieto and David Wilkinson for providing in situ hybridization protocols and advice. N.M. was supported in part by an ISRT fellowship, D.M. by a MRC studentship, and N.G. by long-term E MBO and Dutch Royal Academy of Sciences (KNAW) fellowships. This research was supported by the MRC (UK) and the Erasmus University Rotterdam.

\section{REFERENCES}

Antoniou, M., deBoer, E., Habets, G., and Grosveld, F. (1988). The human beta-globin gene contains multiple regulatory regions: Identification of one promoter and two downstream enhancers. EMBO J . 7: 377- 384.

Attar, R. M., and Gilman, M. Z. (1992). Expression cloning of a novel zinc finger protein that binds to the c-fos serum response element. Mol. Cell. Biol. 12: 2432-2443.

Auffray, C., and Rougeon, F. (1980). Purification of mouse immunoglobulin heavy-chain messenger RNAs from total myeloma tumor RNA. Eur. J . Biochem. 107: 303-314.

Bellefroid, E. J ., Lecocq, P. J ., Benhida, A., Poncelet, D. A., Belayew, A., and Martial, J . A. (1989). The human genome contains hundreds of genes coding for finger proteins of the Kruppel type. DNA 8: $377-387$.

Bellefroid, E. J ., Poncelet, D. A., Lecocq, P. J ., Revelant, O., and Martial, J . A. (1991). The evolutionarily conserved Kruppel-associated box domain defines a subfamily of eukaryotic multifingered proteins. Proc. Natl. Acad. Sci. USA 88: 3608-3612.

Bellefroid, E. J ., Marine, J. C., Ried, T., Lecocq, P. J ., Riviere, M., Amemiya, C., Poncelet, D. A., Coulie, P. G., de J ong, P., Szpirer, C., et al. (1993). Clustered organization of homologous KRAB zincfinger genes with enhanced expression in human T Iymphoid cells. EMBO J . 12: 1363-1374.

Bird, A. P. (1986). CpG-rich islands and the function of DNA methylation. Nature 321: 209-213.

Burke, P. S., and Wolgemuth, D. J . (1992). Zfp-37, a new murine zinc finger encoding gene, is expressed in a developmentally regulated pattern in the male germ line. Nucleic Acids Res. 20: 2827- 2834.

Chowdhury, K., Deutsch, U., and Gruss, P. (1987). A multigene family encoding several "finger" structures is present and differentially active in mammalian genomes. Cell 48: 771- 778.

Decker, C. J ., and Parker, R. (1994). Mechanisms of mRNA degradation in eukaryotes. Trends Biochem. Sci. 19: 336- 340.

Devereux, J ., Haeberli, P., and Smithies, O. (1984). A comprehensive set of sequence analysis programs for the VAX. Nucleic Acids Res. 12: $387-395$.

Fairall, L., Schwabe, J. W., Chapman, L., Finch, J . T., and Rhodes, D. (1993). The crystal structure of a two zinc-finger peptide reveals an extension to the rules for zinc-finger/DNA recognition. Nature 366: $483-487$.

Feinberg, A. P., and Vogelstein, B. (1983). A technique for radiolabeling DNA restriction endonuclease fragments to high specific activity. Anal. Biochem. 132: 6- 13.

Foulkes, N. S., Schlotter, F., Pevet, P., and Sassone-Corsi, P. (1993). Pituitary hormone FSH directs the CREM functional switch during spermatogenesis. Nature 362: 264- 267.

Fourney, R. M., Miyakoshi, J ., Day, R. S., and Paterson, M. C. (1988). Northern blotting: Efficient RNA staining and transfer. Focus 10: 5-7.

Freter, R. R., Irminger, J . C., Porter, J . A., J ones, S. D., and Stiles, C. D. (1992). A novel 7-nucleotide motif located in $3^{\prime}$ untranslated sequences of the immediate-early gene set mediates platelet-derived growth factor induction of the J E gene. Mol. Cell. Biol. 12: 5288-5300.

Frohman, M. A., Dush, M. K., and Martin, G. R. (1988). Rapid production of full-length CDNAs from rare transcripts: Amplification us- ing a single gene-specific oligonucleotide primer. Proc. Natl. Acad. Sci. USA 85: 8998-9002.

Grosveld, F. G., Lund, T., Murray, E. J ., Mellor, A. L., Dahl, H. H., and Flavell, R. A. (1982). The construction of cosmid libraries which can be used to transform eukaryotic cells. Nucleic Acids Res. 10: $6715-6732$.

Harrison, S. C. (1991). A structural taxonomy of DNA-binding domains. Nature 353: 715- 719.

Herrin, D. L., and Schmidt, G. W. (1988). Rapid, reversible staining of Northern blots prior to hybridization. BioTechniques 6: 196197.

Hinnebusch, A. G. (1994). Translational control of GCN4: An in vivo barometer of initiation-factor activity. Trends Biochem. Sci. 19: 409- 414.

Hosseini, R., Marsh, P., Pizzey, J ., Leonard, L., Ruddy, S., Bains, S., and Dudley, K. (1994). Restricted expression of a zinc finger protein in male germ cells. J . Mol. Endocrinol. 13: 157- 165.

Keller, A. D., and Maniatis, T. (1992). Only two of the five zinc fingers of the eukaryotic transcriptional repressor PRDI-BF 1 are required for sequence-specific DNA binding. Mol. Cell. Biol. 12: 1940- 1949.

Koster, M., Kuhn, U., Bouwmeester, T., Nietfeld, W., el-Baradi, T., Knochel, W., and Pieler, T. (1991). Structure, expression and in vitrofunctional characterization of a novel RNA binding zinc finger protein from Xenopus. EMBO J . 10: 3087- 3093.

Margolin, J . F., Friedman, J . R., Meyer, W. K., Vissing, H., Thiesen, H. J ., and Rauscher, F., III. (1994). Kruppel-associated boxes are potent transcriptional repression domains. Proc. Natl. Acad. Sci. USA 91: 4509-4513.

McMahon, A. P., and Bradley, A. (1990). The Wnt-1 (int-1) protooncogene is required for development of a large region of the mouse brain. Cell 62: 1073-1085.

Murphy, G., and Kavanagh, T. (1988). Speeding-up the sequencing of double-stranded DNA. Nucleic Acids Res. 16: 5198.

Nardelli, J ., Gibson, T. J ., Vesque, C., and Charnay, P. (1991). Base sequence discrimination by zinc-finger DNA-binding domains. Nature 349: 175- 178.

Nelki, D., Dudley, K., Cunningham, P., and Akhavan, M. (1990). Cloning and sequencing of a zinc finger cDNA expressed in mouse testis. Nucleic Acids Res. 18: 3655.

Nieto, M. A., Gilardi-Hebenstreit, P., Charnay, P., and Wilkinson, D. G. (1992). A receptor protein tyrosine kinase implicated in the segmental patterning of the hindbrain and mesoderm. Development 116: 1137-1150.

Parkhurst, S. M., Harrison, D. A., Remington, M. P., Spana, C., Kelley, R. L., Coyne, R. S., and Corces, V. G. (1988). The Drosophila $\mathrm{su}(\mathrm{Hw})$ gene, which controls the phenotypic effect of the gypsy transposable element, encodes a putative DNA-binding protein. Genes Dev. 2: 1205-1215.

Pavletich, N. P., and Pabo, C. O. (1991). Zinc finger-DNA recognition: Crystal structure of a Zif268- DNA complex at 2.1 A. Science 252: 809-817.

Pavletich, N. P., and Pabo, C. O. (1993). Crystal structure of a fivefinger GLI-DNA complex: New perspectives on zinc fingers. Science 261: $1701-1707$.

Pengue, G., Calabro, V., Bartoli, P. C., Pagliuca, A., and Lania, L. (1994). Repression of transcriptional activity at a distance by the evolutionarily conserved KRAB domain present in a subfamily of zinc finger proteins. Nucleic Acids Res. 22: 2908- 2914.

Rosati, M., Marino, M., Franze, A., Tramontano, A., and Grimaldi, $\mathrm{G}$. (1991). Members of the zinc finger protein gene family sharing a conserved N-terminal module. Nucleic Acids Res. 19: $5661-$ 5667.

Roseman, R. R., Pirrotta, V., and Geyer, P. K. (1993). The su(Hw) protein insulates expression of the Drosophila melanogaster white gene from chromosomal position-effects. EMBO J . 12: 435-442.

Saiki, R. K., Gelfand, D. H., Stoffel, S., Scharf, S. J., Higuchi, R., 
Horn, G. T., Mullis, K. B., and Erlich, H. A. (1988). Primer-directed enzymatic amplification of DNA with a thermostable DNA polymerase. Science 239: 487-491.

Sambrook, J ., Fritsch, E. F., and Maniatis, T. (1989). "Molecular Cloning: A Laboratory Manual", 2nd ed., Cold Spring Harbor Laboratory Press, Cold Spring Harbor, NY.

Sanger, F., Nicklen, S., and Coulson, A. R. (1977). DNA sequencing with chain-terminating inhibitors. Proc. Natl. Acad. Sci. USA 74: 5463-5467.

Schuh, R., Aicher, W., Gaul, U., Cote, S., Preiss, A., Maier, D., Seifert, E., Nauber, U., Schroder, C., Kemler, R., et al . (1986). A conserved family of nuclear proteins containing structural elements of the finger protein encoded by Kruppel, a Drosophila segmentation gene. Cell 47: 1025-1032.

Theunissen, O., Rudt, F., Guddat, U., Mentzel, H., and Pieler, T. (1992). RNA and DNA binding zinc fingers in Xenopus TFIIIA. Cell 71: 679-690.

Witzgall, R., O'Leary, E., Leaf, A., Onaldi, D., and Bonventre, J . V. (1994). The Kruppel-associated box-A (KRAB-A) domain of zinc finger proteins mediates transcriptional repression. Proc. Natl. Acad. Sci. USA 91: 4514-4518.

Xie, J ., Roddy, P., Rife, T. K., Murad, F., and Young, A. P. (1995). Two closely linked but separable promoters for human neuronal nitric oxide synthase gene transcription. Proc. Natl. Acad. Sci. USA 92: 1242- 1246. 